
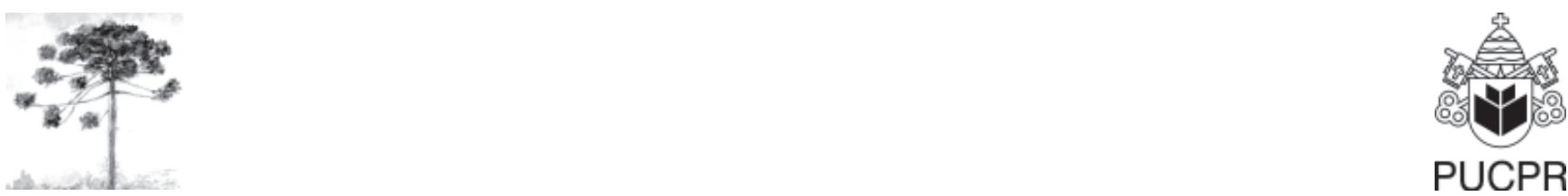

\title{
TALASSEMIA BETA MINOR: estudo de caso e revisão da literatura
}

\author{
Beta minor thalassemia: a case study and literature review
}

\section{Luisandro Lermen ${ }^{[1]}$, Bruno Rizzo Osternack ${ }^{[2]}$, Sabrina Gonçalves ${ }^{[3]}$, Paulo Henrique da Silva ${ }^{[4]}$, Patrícia Haas ${ }^{[5]}$}

${ }^{[1]}$ Laboratório da Prefeitura Municipal de Itapiranga, Itapiranga, SC - Brasil

${ }^{[2]}$ Laboratório do Hospital Erasto Gaertner, Curitiba, PR - Brasil.

${ }^{[3]}$ Mestre em Farmácia-Análises Clínicas, Universidade Federal de Santa Catarina (UFSC), Florianópolis, SC - Brasil.

${ }^{[4]}$ Professor de Hematologia na Universidade Federal do Paraná (UFPR), Curitiba, PR - Brasil.

${ }^{[5]}$ Professora do Departamento de Análises Clínicas da Universidade Federal de Sana Catarina (UFSC), Florianópolis, SC Brasil, e-mail: haas@icb.ufsc.br

\section{Resumo}

As talassemias compreendem os distúrbios genéticos da síntese de hemoglobina caracterizados por redução parcial ou total na produção de uma ou mais cadeias polipeptídicas de globina. A maioria dos heterozigotos para talassemia beta são assintomáticos e desconhecem o defeito genético do qual são portadores. Existe uma deficiência no diagnóstico clínico-laboratorial para a investigação dessa doença que acomete uma parcela significativa da população brasileira. Dessa maneira, o diagnóstico precoce continua sendo um grande instrumento para aumentar a proteção ao portador de talassemia. Os objetivos deste trabalho são investigar uma família da cidade de Itapiranga, SC, quanto à presença do traço talassêmico e realizar uma breve revisão da literatura sobre a importância do diagnóstico precoce e do aconselhamento genético na conscientização dos portadores de anemias hereditárias. É relatado um caso de traço talassêmico em um homem de 42 anos de idade, e a importância do diagnóstico precoce e do aconselhamento genético na conscientização dos portadores de anemias hereditárias é revisado.

Palavras-chave: Anemia hereditária; Talassemia; Diagnóstico precoce; Aconselhamento genético. 


\begin{abstract}
The thalassemia understand the genetic disturbances of the synthesis of hemoglobin characterized by partial reduction or total in the output of an or more chains polipeptídicas of globina. Most heterozygous for beta thalassemia are asymptomatic and are unaware of the genetic defect they carry. There is a deficiency in the laboratorial-clinical diagnosis for the investigation of this disease that affects a significant part of the Brazilian population. This way, the early diagnosis is still a great tool to increase the protection of the thalassemia carrier. The objectives of this work are the of investigate a family of the city of Itapiranga-SC, as regards the presence of the line thalassemia and carry out a short revision of the literature about the importance of the early diagnosis and of the genetic counselling in the awareness of the bearers of hereditary anemias. A case of thalassemia tracing in a 42-years-old is described, and the importance of the early diagnosis and genetic counseling in the awareness of individuals with hereditary anaemia is reviewed.
\end{abstract}

Keywords: Hereditary anaemia; Thalassemia; Early diagnosis; Genetical counseling.

\section{INTRODUÇÃO}

As talassemias compreendem os distúrbios genéticos da síntese de hemoglobina caracterizados por redução parcial ou total na produção de uma ou mais cadeias polipeptídicas de globina. Essa redução provoca um desequilíbrio entre os diferentes tipos de globina, resultando no desenvolvimento de uma anemia microcítica e hipocrômica. De acordo com a cadeia globínica afetada, as talassemias podem ser classificadas como: alfa, beta, delta, delta-beta, e gama-delta-beta. Estima-se que, no mundo, 15 milhões de pessoas sejam talassêmicas $(1,2)$, entretanto, as talassemias alfa e beta são consideradas as mais importantes do ponto de vista da saúde pública, e as mais frequentes em todo o mundo $(3,4)$.

A talassemia beta pode ser causada por mutação em ponto, deleção ou inserção de poucos nucleotídeos, resultando num decréscimo da transcrição, incapacidade de iniciação da tradução, processamento anormal de RNA, matriz de leitura, terminação prematura e produção de hemoglobinas instáveis $(5,6)$. O modo de herança das talassemias é autossômico recessivo, sendo necessários dois genes anormais de globina beta para produzir o fenótipo clinicamente detectável (7).

Em virtude do fluxo de migração das populações, a talassemia beta pode ser encontrada em todos os continentes, estimando-se mais de $100 \mathrm{mil}$ pacientes vivendo com esta doença no mundo (8).

Clinicamente são identificados dois grupos de talassemia beta: talassemia minor (traço talassêmico ou talassemia heterozigota beta) e talassemia major (ou talassemia homozigota beta). Alguns pacientes ficam entre esses dois extremos, sendo considerados portadores da talassemia intermediária (9).

De acordo com um estudo feito em 65 cidades de 16 estados do Brasil, aproximadamente 2,7 milhões de brasileiros são portadores da talassemia minor, a qual não requer tratamento por ser apenas uma característica genética, e não uma doença. Seu portador não apresenta quaisquer sintomas e pode viver com o traço sem nunca saber de sua existência $(10,11)$.

Já os portadores de talassemia major, se não forem tratados, podem apresentar icterícia e esplenomegalia, que se tornam evidentes na primeira infância. Ossos frontais, maxilares e mandíbulas proeminentes dão uma aparência mongolóide. Essas alterações refletem a extrema hiperplasia medular em resposta ao processo hemolítico. O crescimento é interrompido e a puberdade é atrasada. A maioria dos pacientes necessita de transfusões regulares e desenvolvem problemas de acúmulo de ferro (9).

Infelizmente, não existem medidas preventivas contra as mutações que interferem na produção de hemoglobina. Dessa maneira, o diagnóstico precoce continua sendo um grande instrumento para aumentar a proteção ao portador de talassemia mais grave e prolongar sua expectativa de vida e também para detectar o traço talassêmico em casais que desejam ter filhos (2).

Os objetivos deste trabalho são o de investigar uma família da cidade de Itapiranga, SC, quanto à presença do traço talassêmico e realizar uma breve revisão da literatura sobre a importância do diagnóstico precoce e do aconselhamento genético na conscientização dos portadores de anemias hereditárias. 


\section{RELATO DO CASO}

Trata-se de paciente do sexo masculino, 42 anos de idade, oriundo do município de Itapiranga, SC. Em sua história pregressa narrou que seus exames laboratoriais sempre apresentaram anemia. Seu hemograma apresentou alterações significativas em alguns índices hematimétricos, como pode ser visto na Tabela 1.

TABELA 1 - Valores do eritrograma na família do paciente portador de talassemia beta minor

\begin{tabular}{lccccc}
\hline Índices & Paciente & Pai & Irmão & Irmã & Filho \\
\hline ERI (x10 / $\boldsymbol{\mu L}) *^{\text {HCT (\%)* }}$ & 5,02 & 6,10 & 6,14 & 4,97 & 4,88 \\
Hb (g/dL)* & 32,9 & 44,0 & 42,0 & 30,9 & 31,2 \\
VCM (fL)* & 11,2 & 14,4 & 12,5 & 9,0 & 10,0 \\
HCM (pg)* & 65,5 & 72,2 & 68,9 & 62,1 & 63,9 \\
CHCM (g/dL)* & 22,4 & 23,7 & 20,5 & 18,0 & 20,6 \\
RDW (\%)* & 34,2 & 32,8 & 28,8 & 29,0 & 32,2 \\
& 16,0 & 16,4 & 16,2 & 16,4 & 15,5 \\
\hline
\end{tabular}

*Valores de Referência: ERI= Eritrócitos (4,00 - 6,00 x 10\%/ $\mu \mathrm{L}) ; \mathrm{HCT}=$ Hematócrito (35,0 -60,0\%); Hb= Hemoglobina $(11,0-18,0$ $\mathrm{g} / \mathrm{dL}) ; \mathrm{VCM}=$ Volume Corpuscular Médio (80,0 -100,0 fL); HCM= Hemoglobina Celular Média (27,0 - 31,0 pg); CHCM=

Concentração de Hemoglobina Corpuscular Média (33,0 - 35,5 g/dL); RDW= Amplitude da Distribuição dos Eritrócitos (11,5-14,5\%).

A Tabela 2 demonstra a avaliação da morfologia eritrocitária em esfregaço sanguíneo corado. As dosagens de ferro sérico e ferritina do paciente podem ser observadas na Tabela 3 . A eletroforese de hemoglobina apresentou genótipo sugestível de traço talassêmico (Tabela 4). Foi necessário estudo familiar para a confirmação diagnóstica. O pai, irmão, irmã e filho do paciente também apresentaram em seus exames complementares genótipo compatível com talassemia beta minor. Os demais resultados estão representados nas Tabelas 1, 2, 3 e 4.

TABELA 2 - Principais alterações eritrocitárias observadas no esfregaço sanguíneo da família do paciente portador de talassemia beta minor

\begin{tabular}{lllll}
\hline Paciente & Pai & Irmão & Irmã & Filho \\
\hline Anisocitose & Anisocitose & Anisocitose & Anisocitose & Anisocitose \\
Hipocromia & Hipocromia & Hipocromia & Hipocromia & Hipocromia \\
Microcitose & Microcitose & Microcitose & Microcitose & Microcitose \\
Policromasia & Policromasia & Policromasia & Policromasia & Policromasia \\
Ponteado & Ponteado & Ponteado & Ponteado & Ponteado \\
Basófilo & Basófilo & Basófilo & Basófilo & Basófilo \\
Eliptócito & & & Eliptócito & Eliptócito \\
Dacriócito & & Dacriócito & Dacriócito \\
& & & & Anel de Cabot \\
\hline
\end{tabular}

TABELA 3 - Níveis séricos de ferro e ferritina na família do paciente portador de talassemia beta minor

\begin{tabular}{lrrrrr}
\hline Níveis & Paciente & Pai & Irmão & Irmã & \multirow{2}{*}{ Filho } \\
\hline Ferro ( $\mathbf{\mu g} / \mathbf{d L}) *$ & 136,0 & 164,0 & 119,0 & 79,0 & 84,0 \\
Ferritina (ng/mL)** & $1.153,0$ & $1.009,0$ & 601,9 & 314,9 & 160,0 \\
\hline
\end{tabular}

*Valores de Referência (ferro sérico): Homens - 59,0 a 158,0 $\mu \mathrm{g} / \mathrm{dL}$ e Mulheres - 37,0 a 145,0 $\mu \mathrm{g} / \mathrm{dL}$.

**Valores de Referência (ferritina): Homens - 30,0 a 40,0 ng/mL; Mulheres - 13,0 a 150,0 ng/mL e Crianças (4 meses a 16 anos) - 20,0 a 200,0 ng/mL. 
TABELA 4 - Fracionamento eletroforético de hemoglobinas na família do paciente portador de talassemia beta minor

\begin{tabular}{lcrrrr}
\hline Frações & Paciente & Pai & Irmão & Irmã & Filho \\
\hline A1 (\%)* & 92,9 & 90,8 & 92,4 & 91,4 & 92,5 \\
A2 (\%)* & 5,7 & 5,3 & 5,0 & 5,3 & 5,6 \\
f (\%)* & 1,4 & 3,9 & 2,6 & 3,3 & 1,9 \\
\hline
\end{tabular}

*Valores de Referência: Hemoglobina A1 - >= 96,8\%; Hemoglobina A2 - entre 2,2 e 3,2\% e Hemoglobina Fetal - < 0,5\%.

\section{DISCUSSÃO}

Em geral, as talassemias apresentam-se de uma forma benigna, mas em certos casos podem ter evolução comprometedora. Fato este que deve estimular os médicos como um todo, eprincipalmente os hematologistas, a se anteciparem no seu diagnóstico para que se faça um bom acompanhamento do paciente, possibilitando uma melhor qualidade de vida. É muito importante, também, o papel do aconselhamento genético na tentativa de se evitar a concepção de pacientes portadores de síndromes talassêmicas graves. O exame laboratorial de ambos os genitores sempre é conveniente para se estabelecer um diagnóstico preciso, pois o aconselhamento genético não pode estar baseado em hipóteses diagnósticas somente (12).

O paciente de 42 anos do presente caso em estudo apresentou valor de hemoglobina e hematócrito dentro dos valores de referência e no limite inferior, respectivamente. A microcitose (VCM $=65,5 \mathrm{fL})$ e a hipocromia $(\mathrm{HCM}=22,4 \mathrm{pg})$ chamam a atenção neste eritrograma. A hipocromia foi difícil de ser visualizada na extensão sanguínea $(\mathrm{CHCM}=$ 34,2 g/dl), mas ela estava presente porque o HCM está abaixo do valor de referência (27 pg). A hipocromia foi marcada em função do valor do HCM. A anisocitose foi outro achado consistente, pois o RDW foi de 16\%. Este eritrograma pode ser classificado como sendo microcítico e hipocrômico e tendo uma população eritrocitária heterogênea. Para dosagem da ferritina pode ser afastada a possibilidade de anemia ferropriva.

A anemia ferropriva foi afastada, também, pelo fato de este eritrograma apresentar características de hemólise (policromasia) e presença de ponteados basófilos. O ponteado basófilo indica que se tem alguma alteração na síntese de hemoglobina, ou uma mitose acelerada (aumento da atividade da medula óssea) ou dificuldade na síntese de hemoglobina. Este paciente é um caso de talassemia beta heterozigota confirmada pela eletroforese de hemoglobina, onde as Hemoglobinas F e A2 estavam aumentadas.

Este diagnóstico também foi confirmado realizando-se uma investigação na família do paciente quantoà herança da talassemia beta e pode-se observar que os resultados hematimétricos e eletroforéticos foram compatíveis com talassemia beta minor.

Alguns estudos, como de Leoneli et al. (13) e Serra et al. (14) demonstraram a alta prevalência de talassemias encontradas em trabalhos que analisaram indivíduos com história familiar para hemoglobinopatias ou anemia a esclarecer. $\mathrm{O}$ aconselhamento genético para 200 pacientes portadores de â-talassemia, com 421 parentes estudados, demonstrou 188 (45\%) novos casos de traço â-talassêmico (14). A distribuição dos pacientes conforme a presença de história familiar para síndromes anêmicas, em pesquisa realizada por Taneja (12), demonstrou: $6(21,42 \%)$ pacientes com história familiar positiva para síndromes talassêmicas; $2(7,15 \%)$ para síndromes falciformes, e em $15(53,57 \%)$ pacientes não havia registro de história familiar (12, 13, 14).

Branco et al. (15) reforçaram a necessidade da história familiar de todos os pacientes com suspeita de hemoglobinopatia para melhor confirmação do diagnóstico de â talassemia (15). Uma evidência dessa importância é a prevalência de $6,4 \%$ de â-talassemia heterozigótica entre paulistas descendentes não miscigenados de italianos $(16,17)$. Na cidade de Colina, SP, a alta prevalência das talassemias pode ser explicada pela contribuição dada pelos colonizadores italianos que, em 1920, representavam cerca de $60 \%$ dos estrangeiros, situação semelhante ocorreu no Sul do Brasil $(17,18)$.

Em estudo realizado por' Taneja (12), sobre síndromes talassêmicas diagnosticadas no Hospital Universitário em Santa Catarina, no período de 1980 a 2004, observou-se que dos 28 casos de 
síndromes talassêmicas diagnosticadas, 17 (60,70\%) casos eram de â-talassemia minor, cinco $(17,85 \%)$ casos de Talassemia AS-á, dois (7,14\%) casos de Sâ0-talassemia, dois $(7,14 \%)$ casos de Sâttalassemia, um caso $(3,57 \%)$ de $\hat{a}$-talassemia major, e um caso de á- talassemia (3,57\%) (12). Logo, a talassemia beta minor foi a síndrome talassêmica mais frequente na população atendida no Hospital Universitário, corroborando os dados obtidos entre os resultados obtidos no estado de São Paulo.

Um casal em que ambos sejam portadores de talassemia beta minor há probabilidade de 25\% de gerar um filho portador de talassemia beta major. Por isso, o exame para detectar o traço talassêmico é fundamental para o casal que deseja ter filhos, para que conheçam a possibilidade de terem um filho com talassemia major, e no caso desta possibilidade se concretizar, saibam que seu filho pode levar uma vida normal como qualquer outra criança, desde que receba o tratamento adequado desde o início $(19,11)$.

As talassemias beta são mais difíceis de serem diagnosticadas do que as outras alterações da hemoglobina. Por essa razão, o diagnóstico normalmente é baseado nos padrões hereditários e em exames especiais da hemoglobina (20). Além disso, os achados laboratoriais da talassemia beta minorsão muito semelhantes aos da anemia por deficiência de ferro e, em alguns casos são adotadas condutas errôneas no tratamento do paciente, como a administração de compostos à base de ferro. Os pacientes com traço talassêmico cursam com maior absorção do ferro no intestino do que as pessoas não talassêmicas. Como a absorção de ferro pode estar aumentada e como transfusões sanguíneas (fornecendo mais ferro) são necessárias, o ferro em excesso pode acumular e depositar-senomiocárdio (músculo cardíaco), podendo acarretar insuficiência cardíaca (21).

Diante do conjunto de resultados e da situação relatada no presente trabalho, conclui-se, primeiramente, que é de fundamental importância o diagnóstico precoce das anemias hereditárias, por meio da determinação dos valores hematimétricos, análises da morfologia eritrocitária e caracterização de genótipos de hemoglobinas variantes. Adicionalmente, é necessário que programas de prevenção sejam repensados, pelo aconselhamento genético e estudo familiar, pois colaboram para a conscientização dos heterozigotos, fornecendo informações para que possam decidir responsavelmente sobre o futuro de seus descendentes $(22,23)$. E, por fim, também se espera que as anemias hereditárias se tornem mais divulgadas entre os profissionais da saúde para que, então, ocorra o aprofundamento do estudo do hemograma nos laboratórios.

\section{REFERÊNCIAS}

1. Clarke GM, Higgins TN. Laboratory investigation of hemoglobinopathies and thalassemias: Clin Chem. 2000;46(8 Pt 2):1284-90.

2. Fleury. Talassemia. [citado 10 de out. 2007. Disponível em http://www.fleury.com.br/ Clientes/SaudeDia.

3. Wagner SC, Silvestri MC, Bittar CM, Friedrisch JR, Silla ML. Prevalência de talassemias e hemoglobinas variantes em pacientes com anemia não ferropênica. Rev Bras Hematol Hemot. 2005;27(1):7-42.

4. Weatherall DJ, Clegg JB. Inherited haemoglobin disorders: an increasing global health problem. Bull World Health Organ. 2001;79(8):704-12.

5. Hattori Y. Globin gene mutation is a model of genetic abnormalities. Rinsho Byori. 1999;47(3):244-51.

6. Lorenzi TF. Manual de hematologia. propedêutica e clínica. São Paulo: Medsi; 1999.

7. Carvalho LB. Avaliação da expressão da talassemia do tipo beta no Brasil pela co-herança com defeitos de hemocromatose São José do Rio Preto 2003. [dissertação]. São Paulo: Universidade Estadual Paulista - UNESP; 2003.

8. Capellini N, Cohen A, Eleftheriou A, Piga A, Porter J, Taher A., et al. Guidelines for the clinical management of thalassaemia. Thalassaemia International Federation, [cited 2009 Dec 1]. Available from: http:/ / www.thalassaemia.org.cy/ pdf/Guidelines_2nd_revised_edition_EN.pdf

9. Elghetany MT, Davey FR. Doenças eritrocitárias. In: Henry J. Diagnósticos clínicos e tratamento por métodos laboratoriais pp. 617718. São Paulo: Manole; 1999.

10. Reis PRM, Penna KGBD, Araújo LMM, Mesquita MM, Castro FS, Balestra FA. Prevalência de hemoglobinopatias e talassemias em crianças de 6 meses a 7 anos de idade no laboratório escola do departamento de biomedicina (CBB)-UCG. Rev Bras Anál Clín. 2005;37(1):3-5. 
11. Steagall, M. Talassemia. [citado 10 out. 2007]. Disponível em: http:/ / republica.com.br/noticias/

12. Taneja AK. Síndromes Talassêmicas Diagnosticadas no Hospital Universitário - 1980 a 2004 [monografia]. Florianópolis: Universidade Federal de Santa Catarina; 2005.

13. Leoneli GG, Imperial RE, Marchi-Salvador DP, Naoum PC, Bonini-Domingos CR. Hemoglobinas anormais e dificuldade diagnóstica. Rev Bras Hemat Hemot. 2000;22(3):396-403.

14. Serra HG, Martins CSB, Paiva e Silva RB, Ramalho AS. Evaluation of genetic counseling offered to Brazilian carriers of the betathalassemia trait and to their relatives. Brazilian Journal of Genetics. 1995;18(3);479-84.

15. Branco RM, Hirano ZMB, Haas LJ, Rosa P. Prevalência de betatalassemia em pacientes portadores de anemia microcítica em Blumenau-SC. Ars Cvrandi. 1999;32:37-40.

16. Ramalho AS. Magna LA, Costa FF, Grotto HZW. Talassemia menor: um problema de saúde pública no Brasil? Rev Brasil Genet. 1985;8(4):747-54.

17. Ramalho AS. A talassemia minor como causa de anemia no estado de SP. Rev Bras Pat Clin. 1986;22(2):32-8.

18. Filho FM, Naoum PC, Moreira HW, Angulo IL. Variabilidade polimórfica de hemoglobinas humanas anormais em indivíduos das cidades de Barretos e Colina, SP, Brasil. Rev Bras Pat Clin. 1988;24(2):32-9.
19. Melo-Reis PR, Araújo LMM, Dias-Penna KGB, Mesquita MM, Castro FS, Costa SHN. A importância do diagnóstico precoce na prevenção das anemias hereditárias. Rev Bras Hematol Hemot. 2006;28(2):149-52.

20. Melo-Reis PR, Naoum PC, Diniz-Filho JAF, Dias-Penna KGB, Mesquita MM, Balestra FA, et al. Prevalência de talassemias e hemoglobinas variantes no estado de Goiás, Brasil. J Bras Patol Med Lab. 2006;42(6):425-30.

21. Failace R, Antunes C. O hemograma no Cell Dyn 4000 no diagnóstico diferencial entre beta talassemia minor e anemia ferropênica. Laes \& Haes. 2003;2:98-114.

22. Compri MB, Polimeno NC, Stella MB, Ramalho AS. Programa comunitário de hemoglobinopatias hereditárias em população estudantil brasileira. Rev Saúde Pública. 1996;30(2):187-95.

23. Orlando GB, Naoum PC, Siqueira FAM, Bonini-Domingos CR. Diagnóstico laboratorial de hemoglobinopatias em populações diferenciadas. Rev Bras Hemat Hemot. 2000;22(2):111-21.
Recebido: 21/09/2007

Received: 09/21/2007

Aprovado: 20/12/2007

Approved: $12 / 20 / 2007$ 